\title{
Nanoengineering of Materials for Field Emission Display Technologies
}

S. R. P. Silva*, J. D. Carey, G. Y. Chen, D. C. Cox, R. D. Forrest, C. H. Poa,

R. C. Smith, Y. F. Tang and J. M. Shannon

Advanced Technology Institute, School of Electronics and Physical Sciences,

University of Surrey, Guildford GU2 7XH, U.K.

The holy-grail in terms of flat panel displays has been an inexpensive process for the production of large area 'hang on the wall' television that is based on an emissive technology. As such electron field emission displays, in principle, should be able to give high quality pictures, with good colour saturation, and, if suitable technologies for the production of the cathodes over large areas were to be made available, at low cost. This requires a process technology where temperatures must be maintained below $450{ }^{\circ} \mathrm{C}$ throughout the entire production cycle to be consistent with the softening temperature of display glass. In this paper we show three possible routes for nanoscale engineering of large area cathodes using low temperature processing that can be integrated into a display technology. The first process is based on carbon nanotube-polymer composites that can be screen printed over large areas and show electron field emission properties comparable with some of the best aligned nanotube arrays. The second process is based on the direct large area growth of carbon nanofibres directly on to substrates held at temperatures ranging from room temperature to $300{ }^{\circ} \mathrm{C}$, thereby making it possible to use inexpensive substrates. The third process is based on the use of excimer laser processing of amorphous silicon for the production of lithography free large area three terminal nanocrystalline silicon substrates. Each route has its own advantages, and flexibility in terms of incorporation into an existing display technology. The harnessing of these synergies will be highlighted together, with the properties of the cathodes developed for the differing technologies.

*Email: s.silva@surrey.ac.uk 


\section{Introduction}

The flat panel display (FPD) market is one of the largest consumer electronic sectors, with sales within the US alone exceeding one billion dollars per annum. There are many competing FPD technologies for the flat displays, as shown in Fig. 1, with active matrix liquid crystal displays (AMLCDs) leading the way. For the larger flat displays, plasma display panels (PDPs) dominate, however, recent developments by Samsung have seen the emergence of AMLCDs with 52 inch diagonal screens. Samsung have also produced a prototype 38 inch full colour video rate carbon nanotube (CNT) display which shows all the positive attributes associated with field emission such as high brightness, high contrast, excellent viewing angles, low power consumption and large area. Other field emission display (FED) technologies based on metal 'Spindt' tips favoured by companies such as Candescent, Pixtech and Motorola have all delivered high quality displays. The UK based company, Printable Field Emitters, have opted for a screen printed graphite-silicon dioxide binder cathode to make their large area displays. Canon, Toshiba, Noritake, MEW and Sony all have their own field emission (FE) based technologies currently being developed for different segments of the market in this fast moving sector. All these companies see the merits associated with having a fully scalable FED technology, but need the cost of production to be lowered in order to enter the consumer market. Other emerging display technologies vying for honours in this sector include polymer and organic light emitting diodes (OLEDs), with no one technology being able to show all the attributes needed for a high quality large flat display that can be produced at a suitable cost and scale. [1]

FEDs operate in a manner which is a hybrid of the AMLCD and the PDP. The addressing of the picture elements is based on the matrix address system developed for AMLCDs, with the emissive display component showing similarities to the PDP output. Hundreds of multiply gated matrix addressed field emission cathodes emit electrons that hit a single pixel, whose brightness to a first order is controlled by the acceleration voltage applied between the cathode and the phosphor anode. The key physical parameters of importance in selecting a suitable cathode material for such an application are, in addition to its longevity, robustness and an ability to readily integrate into a 
production process, the requirement of being able to source high current densities at relatively low electric fields. In addition, an ability to produce uniform electron emission current-voltage characteristics with little or no hysteresis is also required. This tightness of the electron emission curves with applied field is important in being able to design matrix driver strategies with the required precision, where suitable offset voltages can be used for turning on gated cathodes. In terms of phosphors, standard high and medium voltage phosphors are at present preferred over the low voltage variety due to the reliability and testing that has been performed in both the CRT arena and plasma displays.

The results presented in this paper are based on three competing strands, all working towards a common goal of affordable large area FED cathodes. The first technology is associated with the production of inexpensive cathodes based on mixing CNT-polystyrene (PS) composites that are castable or screen printable over large areas. [2] Their performance as an electron source is tested against other competing nanotube (NT) emitters, as well as Spindt tips. The key marketable feature of CNTcomposite emitters are reliability and costs, which compare favourably with the performance of other more expensive and complicated cathodes. We show the current density and threshold fields afforded by these cathodes are some of the best available in the published literature.

The second technology introduced in this paper is associated with the availability of a low temperature growth process for the production of carbon nanotubes and carbon nanofibres (CNFs). [3] In this case we show the structure of the deposited material is ideally suited for electron field emission, and due to the very low temperature plasma based growth process, can be easily incorporated into a large area display technology. We highlight the advantages associated with a direct in-situ cold cathode growth environment, that gives nanometre precision in the CNT/CNF growth due to the requirement of a suitable catalyst, with the correct dimensions. [4] Due to the low growth temperature the requirement of a barrier layer between the catalyst and the substrate to prevent the transition metal from diffusing may be relaxed. The plasma based growth process is also scalable to very large areas in an inexpensive manner, and can grow materials on non-uniform and non-conformal surfaces such as 
gated cathode tracks due to the catalytic plasma based growth process. The technology also lends itself to domestic lighting applications. [5]

The third technology is based on nano-silicon field emitters and was originally introduced in order to exploit a holistic approach to the production of FEDs. The idea being that as amorphous silicon and polycrystalline silicon thin film transistors (TFTs) were already being prepared over very large areas in AMLCDs, if the electron emission component of the FED could also be produced using the same material it would allow a seamless transition of the thin film silicon fabrication facilities to a FED technology. We show how amorphous silicon excimer laser crystallised over large areas can be used as a cathode material. The excimer laser treatment, at low energy densities, affects only the top few nanometres of the $a-\mathrm{Si}: \mathrm{H}$ layer and produces nanocrystalline structures, which would give rise to the enhanced emission properties. This process is then extended to produce lithography free three terminal field emission structures using large area processes that lend itself to fully integrated low cost thin film silicon based cold cathode technology.

\section{Electron field induced emission}

Field emission is the extraction of electrons from a surface under the influence of an applied electric field. The front surface potential barrier for electron emission is reduced by the application of voltage $\mathrm{V}_{\mathrm{a}}$ to an anode located at a distance $\mathrm{D}$ away. Far from the emitter surface the macroscopic field is simply $\mathrm{V}_{\mathrm{a}} / \mathrm{D}$. For tip based structures this macroscopic field is enhanced in the neighbourhood of an emitter by a geometric field enhancement factor, $\beta$. This results in a local electric field which is larger than the applied field. The most common definition of the field enhancement factor, $\beta$, is the ratio of the local field to the applied field. In the case of an isolated vertically aligned CNT in the electrode geometry presented in Fig. 2(a), the local field depends on the height $h$, radius $r$ and anode-substrate separation D. Shown in Fig. 2(b) are the results of a 2D electrostatic simulation for the variation of $\beta$ with $\mathrm{D}$ for a three metallic tubes of heights 2,4 and $6 \mu \mathrm{m}$ capped with a hemisphere. It is apparent that the enhancement factor in this case is only constant (i.e. independent of the electrode geometry) 
when $\mathrm{D}>(2-3) h$. As a result care must be taken in analysing FE measurements on electrode geometries similar to the one described in Fig. 2(a) to ensure that the anode is sufficiently far away for the emitter for this effect to be ignored. The discussion above is based on a single isolated emitter. When there are a large number of emitters nearby screening of the applied field can occur. This has been observed experimentally by Nilson et al. who concluded that screening is an important factor when the intertube separation is less than twice the nanotube length. [6]

In terms of experimental method, FE measurements may be conducted in a number of ways. In this paper two common methods are employed. The first utilises a sphere-to-plane geometry with a $5 \mathrm{~mm}$ stainless steel ball bearing suspended $30 \mu \mathrm{m}$ above the cathode surface with a high positive potential applied in a vacuum better than $4 \times 10^{-6} \mathrm{mbar}$. Although a spherical anode is used it is assumed that the electric field between the anode and the surface of the film can be modelled as a parallel plate. The probe anode is attached to a high precision motor allowing FE measurements to be made for varying anode-substrate distance. The threshold field, $E_{\text {th }}$ is defined as the macroscopic electric field which gives an emission current of $1 \mathrm{nA}$. It has sometimes been observed that there are differences between the upward and downward I-E cycles and that sometimes a large initial field needs to be applied. This is referred to as 'conditioning' of the emitter and tends to be present in less conductive materials such amorphous carbon thin films. [7] As a measure of this "hysteresis effect" the difference in the applied fields between the upward and downward voltage cycles at a current of $1 \mathrm{nA}$ is used. The second way of performing FE measurements uses a planar anode usually a transparent conducting oxide onto which phosphor has been coated to allow emission site density measurements to be made.

\section{Carbon Nanotube-Polymer Composite Cathodes}

Multiwalled carbon nanotubes (MWNTs) and boron-doped MWNTs (B-MWNTs) were produced by the arc discharge method. [2] In the case of B-MWNTs boron containing electrodes were used. The resultant tubes were then purified using the vacuum-filtering technique and were then oxidized at 500- 
$600{ }^{\circ} \mathrm{C}$ for $40-50 \mathrm{~min}$ to remove the amorphous carbon and carbon particles as described elsewhere [2]. Transmission electron microscope images of the purified tubes showed that about $90 \%$ of the tubes have diameters in the $10-50 \mathrm{~nm}$ range. By mechanically bending the film to produce a $4 \mu \mathrm{m}$ fracture it is possible to allow some of the tubes to appear out of the composite as shown in Fig. 3(a). Electron energy loss spectroscopy indicates that the B concentration is about 1 at. \%. Nanotube polymer composites were prepared by mixing with polystyrene and toluene and using vacuum casting techniques. The as-cast films were hot pressed to remove residual solvent and prevent voids in the films as described elsewhere. [2] The variation of film's surface resistivity with nanotube loading is shown in Fig. 3(b) for both doped and undoped NTs where it can be observed that the sheet resistivity of the doped composite falls from $2.5 \Omega \mathrm{cm}$ to $5 \times 10^{-4} \Omega \mathrm{cm}$ as the nanotube loading increases from $11 \%$ to $33 \%$. Over the same nanotube loading range the sheet resistivity of the undoped composite film falls from 15 to $0.1 \Omega \mathrm{cm}$. The film resistivity decreases as the CNT mass fraction increases which indicates that the electrical conduction across the film is mainly controlled by the CNT network. The lower sheet resistivity of the B containing film is attributed the presence of B doping.

The variation of the threshold field for emission measured using a probe anode with the CNT concentration is shown in Fig. 3(c) where it is apparent that the threshold field increases with the CNT content for both MWNT- and B-MWNT-PS films. Despite the B-MWNT being more conductive (as inferred from the sheet resistivity measurements) there is no significant difference of $E_{\text {th }}$ compared with the undoped MWNT. Indeed, it can also be observed that the threshold field of the doped film is higher than that of the undoped film at low nanotube concentrations. The increase in threshold field with CNT content is probably due to the field screening effects as described previously at the composite surface.

The FE characteristics measured using the planar set up are shown for the undoped and doped composites in Fig. 4 (a) and (b). The threshold fields measured are similar to those obtained from the single probe method. The current density is determined across a fixed test area of $5 \mathrm{~mm}^{2}$ and the emission area is controlled by the shape of polytetrafluoroethylene spacer during the experiment. All of 
the films show current saturation at the high current density of about $4 \times 10^{-4} \mathrm{~A} / \mathrm{cm}^{2}$. The origin of the current saturation effect is unclear and has been attributed to several sources such as conduction limited transport in the cathode [8], contact resistance [9], vacuum space-charge effects, [10] and to the effect of absorbates atoms on the CNT surface [11]. However, for a typical field emitter with a work function of $5 \mathrm{eV}$ and an estimated vacuum space-charge emission current density of $10^{8} \mathrm{~A} / \mathrm{cm}^{2}$, then the saturation current for a single CNT with an emission area of $100 \mathrm{~nm}^{2}$ would occur at a current of $10^{-4}$ A. Based on the current saturation levels shown in Fig. 4, which are the limiting values discussed for a single emitter, vacuum space-charge effects appear to be unlikely in this case where multiple emitters are present. The role of energy balance between the apex and body of the emitter as well as dynamic thermal effects such as the Nottingham cooling effect may also need to be taken into consideration. [12]

The emission site density maps for a $3 \mathrm{~mm}^{2}$ surface area of B doped MWNT with $20 \%$ nanotube loading in PS is shown in Fig. 5. It can be seen that the emission across the phosphor plate increases as the applied field increases from $5 \mathrm{~V} / \mu \mathrm{m}$ to $8 \mathrm{~V} / \mu \mathrm{m}$. The estimated emitter site density is $1.3 \times 10^{7} \mathrm{~cm}^{-2}$. Figure 6 shows the variation of emission current over a duration of 50 hours for a $20 \%$ MWNT-PS film. The current density was measured before and after current saturation effect at $0.8 \mathrm{~mA} / \mathrm{cm}^{2}$ and $1.2 \mathrm{~mA} / \mathrm{cm}^{2}$ respectively. Despite slight variations initially for a current density of 1.2 $\mathrm{mA} / \mathrm{cm}^{2}$, stable emission has been observed over the $50 \mathrm{hr}$ duration. Such a value of current density is suitable for a FED structure.

\section{Low Temperature Nanostructured Carbon Cathodes}

There is a general agreement that the growth mechanism for CNT growth proceeds with $\mathrm{C}$ adsorption on to the surface of a nm-sized Ni catalyst (usually in the form of near spherical islands) followed by bulk diffusion of $\mathrm{C}$ through the $\mathrm{Ni}$ forming a supersaturated solution from which carbon precipitates. [13] The Ni is raised from the substrate itself which is energetically favourable especially for small diameter islands - this is known as the 'tip' growth mechanism. One disadvantage of using Si as a 
substrate, especially at high temperature for which CNT growth is usually performed, is that a Ni silicide can be formed and as a result there has been considerable effort in using alternative oxide substrates onto which $\mathrm{Ni}$ is deposited. [14] By growing CNF (which are larger diameter CNTs) at lower temperatures, the formation of the silicide is largely suppressed. The drive to lower temperatures has also resulted in the study of other ways to produce nm sized Ni structures. One such way is to use plasma treatment of Ni thin films.

Carbon nanofibres (CNFs) were grown on Ni thick films which has previously subjected to an Ar ion plasma. The Ar plasma treatment of the Ni film produces a roughening of the film surface and the appearance of circular features of diameters between $3 \mu \mathrm{m}$ and $500 \mathrm{~nm}$ in diameter. Nanofibre growth was then achieved using $\mathrm{CH}_{4}$ as a source gas at a pressure of 1 Torr. The reverse power of the plasma was continually adjusted to keep it as close to zero as possible in order to prevent substantial substrate heating. The water cooled substrate table temperature was monitored to be about $30^{\circ} \mathrm{C}$ throughout the deposition. A SEM image of the surface after growth, shows fibre-like material arranged radially in clusters forming star shaped objects, approximately $50 \mu \mathrm{m}$. The nanofibres are predominantly lying on the surface rather than vertically aligned and towards the centre of the cluster a higher concentration of fibres, with diameters of $100-400 \mathrm{~nm}$, can be seen in Fig. 7(a). No evidence of metal particles in the tips of the CNFs is observed suggesting that the growth does not proceed in a 'tip-based' growth process. The FE characteristics of the CNFs were examined using a sphere-toplane probe testing system. The film deposited at room temperature displayed excellent emission characteristics with a threshold field of $5 \mathrm{~V} / \mu \mathrm{m}$ as shown in Fig. 7(b). The first two current-voltage characteristics are presented and no significant difference between the two I-E characteristics can be seen. Further voltage cycles show the same behaviour and implies that there is no need for a “conditioning" phase. In addition, there is no evidence of hysteresis behaviour between the upward and downward cycle of either I-E characteristic. To exclude the possibility of emission from the roughened $\mathrm{Ni}$ film substrate, or from the surrounding amorphous carbon film, the emission characteristics from these two samples were also tested. In the case of the former only background 
noise was observed and from the latter a threshold field $20 \mathrm{~V} / \mu \mathrm{m}$ was required for emission. This preliminary study shows promising FE characteristics are possible from low temperature grown CNFs.

\section{Nanosilicon based material cathodes}

In the realms of large area FEDs, the possibility of exploiting silicon based materials as the electron source has a number of significant advantages. Silicon, especially the amorphous and polycrystalline forms are now well established as the preferred choice in industry for display driver electronics as well as in other large area electronic applications such as photovoltaic devices. In recent times the ability to produce micro- or polycrystalline silicon over large areas has negated the need to use large area and expensive single crystalline silicon as substrates. Whilst FE from tip based crystalline silicon has led to silicon field emitters arrays (FEAs) [15] the problems associated with the scalability of this emitter system has contributed to preventing this cathode system being exploited in FPDs. Indeed many of the advantages of silicon that are often quoted to justify its use as a FEA material, such as excellent electronic properties, ready availability coupled with a well developed growth and fabrication process can equally be applied to micro- and poly-crystalline Si. An early study [16] of FE from PECVD grown $a$-Si:H showed that an initial conditioning process was required before the onset of stable and reproducible emission could be observed. After conditioning a threshold field of about $5 \mathrm{~V} / \mu \mathrm{m}$ was measured. These films have a low defect density $\left(10^{16} \mathrm{~cm}^{-3}\right)$ and possess about 10 at. $\% \mathrm{H}$ present and as such are similar to those found in device applications such as the switching elements in matrix addressed screens. Lifetime tests made on $a$-Si:H cathodes demonstrated that for a matrix line addressed image with a frame time of $20 \mathrm{~ms}$, a lifetime of 25,000 hours was possible. [16] The mechanism of emission proposed was based on the introduction of hot electrons from the substrate into the conduction band of the amorphous silicon film which are then accelerated towards the front surface due to a combination of depletion field at the back contact and the high degree of field penetration found in this low defect density material. [16] 
One of the principal disadvantages of $a-\mathrm{Si}: \mathrm{H}$ thin films is the relatively low carrier mobility. As a consequence effort has been employed to improve the electrical properties of amorphous silicon based materials. Thermal furnace annealing for several hours at high temperatures has been employed but the temperatures usually used are often above the softening temperature of the typical soda glass used in displays. An alternative is to perform rapid thermal annealing in which the glass substrate is held at a high temperature for a short time such that softening does not become a significant factor. One disadvantage of RTA is that the grain size of the resultant film is smaller than that found after furnace annealing. Furthermore, both types of annealing result in modification of the whole thin film, as a result alternative techniques which allows controlled modification, ideally on a nanometre scale, of the region near the film surface are attractive. One such technique is the use of laser crystallisation in which the large uniform beam size coupled with a suitable choice of wavelength allows large area modification but in which the high transfer of energy takes place within a narrow nm-sized region near the surface. Laser crystallisation of amorphous silicon has been performed using both excimer laser excitation [17] at $248 \mathrm{~nm}$ and a frequency doubled Nd:YAG laser operating at $532 \mathrm{~nm}$. [18] Both the grain size and surface rms roughness can be controlled by a proper combination of the energy density and/or number of pulses employed. FE measurements made on excimer laser crystallised $a$-Si:H films at an energy density of $204 \mathrm{~mJ} / \mathrm{cm}^{2}$ showed that a threshold field for emission of about $15 \mathrm{~V} / \mu \mathrm{m}$ was required. It was reported that when the emission was imaged on a phosphor plate at a field of 30 $\mathrm{V} / \mu \mathrm{m}$, the site density coverage was in excess of $80 \%$. Individual emission sites could not be imaged as the emission observed was uniformly bright throughout except for 2 or 3 very high brightness regions. Significantly, it was observed that no conditioning stage was required for the onset of stable emission and that the hysteresis in the upward and downward voltage cycle is less than $1 \mathrm{~V} / \mu \mathrm{m}$. A careful study of the microstructure of the crystallized film using transmission electron microscope images showed evidence of columnar Si nanocrystals around $90 \mathrm{~nm}$ in height and around $50 \mathrm{~nm}$ in diameter, surrounded by grain boundaries. The observation of FE at an applied field of $15 \mathrm{~V} / \mu \mathrm{m}$ compares favorably with that of emission from Si nanowires $15 \mathrm{~V} / \mu \mathrm{m}$ [19], from poly-Si by 
LPCVD with oxidation sharpening [20] and poly-Si microtips [21] at $~ 20 \mathrm{~V} / \mu \mathrm{m}$ as well as from single $c$-Si microtips [22] at around $20 \mathrm{~V} / \mu \mathrm{m}$.

Laser crystallisation of $a$-Si:H has also been applied to produce a self-aligned microtip array without the need for complex lithography or processing.[23] Using a excimer KrF laser at an energy density of $242 \mathrm{~mJ} / \mathrm{cm}^{2}$ crystallised strips of films $4 \mathrm{~mm}$ wide were produced. This crystallised area was then capped with a $150 \mathrm{~nm}$ thick $a$-SiN layer followed by a $25 \mathrm{~nm}$ layer of evaporated $\mathrm{Cr}$ as schematically shown in Fig. 8(a). Atomic force microscope image (Fig. 8(b)) of the surface shows that surface is uniformly covered in a densely packed irregular array of spheroid structures about $300 \mathrm{~nm}$ in height and an estimated emitter density of $10^{8}-10^{9} \mathrm{~cm}^{-2}$. The crystallised film was then subjected to a rf/microwave reactive ion $\left(\mathrm{CF}_{4} / \mathrm{Ar}\right)$ etch treatment of $200 \mathrm{~W}$ rf power, $900 \mathrm{~W}$ microwave power. It was observed that etching for different times up to 14 mins resulted in the successive etching of the $\mathrm{Cr}$ and SiN layers and the emergence of the Si tip with the tip level with the Cr rim as shown in Fig. 9. Etching for longer times (21 mins) results in the Si tip lying below the Cr rim as shown in Fig. 9(d). However, whilst the self-aligned array structure is obvious, breaks in the gate insulator can be observed for this etching time. For this structure to be of practical use, control of the leakage current as well as a balance between etching of the gate rim material and optimum height of the tip material needs to be found.

\section{Conclusions}

In conclusion, we have described and characterised three potential cathode systems that could be used in the next generation of field emission flat panel display. We have shown that by engineering the cathodes at a nanometer level it is possible to tailor the emission characteristics. For CNT-polymer composite the ability to adjust the nanotube concentration allows us to control the threshold field and emission site density. Carbon nanofibre emitters grown at substrate temperatures close to room temperature open up a new avenue for FED cathodes. Finally laser crystallisation of amorphous silicon has been shown to produce nanometer sized self-aligned gated structures. All these techniques 
offer unique advantages over each other and are suitable for scale up to a meter and beyond. The technology associated with field emission displays associated with FEDs is currently available, however, the commercial viability and market impact remain to be established.

\section{Acknowledgments}

The authors would like to thank the EPSRC for funding through Portfolio Partnership Award and through the Carbon Based electronics programmes. One of the authors (JDC) also acknowledges the EPSRC for an Advanced Research Fellowship. The authors are also grateful to Prof. H W Kroto and the group at the University of Sussex for the development of the CNT-polymer composites as part of a joint EPSRC programme. 


\section{References}

1 See, for example, the Proceedings of the International Vacuum Microelectronics Conference, published in the Journal of Vacuum Science and Technology or the Journal of Society for Information Display.

2 P. C. P. Watts, W. K. Hsu, G. Z. Chen, D. J. Fray, H. W. Kroto, and D. R. M. Walton, J. Mater. Chem. 11, 2482 (2001).

3 B. O. Boskovic, V. Stolojan, R. U. A. Khan, S. Haq and S. R.P. Silva, Nature Materials 1, 165 (2002).

4 V. I. Merkulov, D. H. Lowndes, Y.Y. Wei, G. Eres and E.Voelkl, Appl. Phys. Lett. 76, 3555 (2000).

5 J.M. Bonard, T. Stockli, O. Noury and A. Chatelain, Appl. Phys. Lett. 78, 2775 (2001).

6 L. Nilsson, O. Groening, C. Emmernegger, O. Kuettel, E. Schaller, L. Sclapbach, H. Kind, J.-M. Bonard and K. Kern, Appl. Phys. Lett. 76, 2071 (2000).

7 J.D. Carey and S.R.P. Silva, Appl. Phys. Lett. 78, 347 (2001).

8 J.B. Cui, K.B.K. Teo, J.T.J. Tsai, J. Robertson, and W.I. Milne, Appl. Phys. Lett. 77, 1831 (2000).

9 J. P. Barour, W. W. Dolan, J. K. Tron, E. E. Martin, and W. P. Dyke, Phys. Rev. 92, 45 (1953).

10 W. A. Anderson, J. Vac. Sci. Technol. B 11, 383 (1993).

11 K. A. Dean and B. R. Chalamala, Appl. Phys. Lett. 76, 375 (2000).

12 C.H. Poa et al. unpublished.

13 M. Chhowalla, K. B. K. Teo, C. Ducati, N. L. Rupesinghe, G. A. J. Amaratunga, A. C. Ferrari, D. Roy, J. Robertson, and W. I. Milne, J. Appl. Phys. 90, 5308 (2001).

14 J. D. Carey, L.L. Ong and S.R.P. Silva, Nanotechnology 14, 1223 (2003).

15 See for example, J. Shaw and J. Itoh, Vacuum Microelectronics, John Wiley \& Sons, Inc. (New York) 2001.

16 S.R.P. Silva, R.D. Forrest and J.M. Shannon, J. Non-Cryst. Solids 230, 1101 (1998).

17 C. T. Angelis, C. A. Dimitriadis, M. Miyasaka, F. V. Farmakis, G. Kamarinos, J. Brini, and J. Stoemenos, J. Appl. Phys. 86, 4600 (1999). 
18 Y.F. Tang, S.R.P. Silva and M.J. Rose, Appl. Phys. Lett. 78, 186 (2001).

19 F. C. K. Au, K. W. Wong, Y. H. Tang, Y. F. Zhang, I. Bello, and S. T. Lee, Appl. Phys. Lett. 75, 1700 (1999).

20 A. A. Evtukh, Thin Solid Films 337, 261 (1999).

21 S. E. Huq, G. H. Grayer, and P. D. Prewett, J. Vac. Sci. Technol. B 15, 2855 (1997).

22 T. Sugino, S. Kawasaki, K. Tanioka, and J. Shirafuji, Appl. Phys. Lett. 71, 2704 (1997).

23 R. D. Forrest, D. C. Cox, Y. F. Tang, J. M. Shannon, and S. R. P. Silva, J. Vac. Sci. Technol. B 21, $1560(2003)$. 


\section{Figure Captions}

Figure 1. Generalised classification of displays. The key to the figure is: DMD - Digital micromirror display, CRT - Cathode ray tube, LCD - Liquid crystal display, FPD - Flat panel display, TFT - Thin film transistor, MOS - Metal-oxide-semiconductor, FLC - Ferroelectric crystal, PDLC - Polymer dispersed liquid crystal and OLED - Organic light emitting diodes.

Figure 2 (a) Electrode geometry of a grounded nanotube of height $h$ and radius $r$ with a substrateanode separation D. The substrate and nanotube represent a grounded equipotential surface with a potential $V_{a}$ applied to the anode. Far from the nanotube the electric field, $E_{0}$, is $V_{a} / D$. (b) Simulated value of the ratio of the field at the tip of the nanotube to $\mathrm{E}_{0}$ - 'field enhancement factor' as a function of $\mathrm{D}$ for three tubes of length $2 \mu \mathrm{m}(\mathbf{\square}), 4 \mu \mathrm{m}(\mathrm{O})$ and $6 \mu \mathrm{m}(\mathbf{\Delta})$.

Figure 3 (a) High resolution scanning electron microscope image of a B doped MWNT - PS composite with $25 \mathrm{wt} \%$ nanotubes present mechanically fractured revealing aligned nanotubes. (b) Sheet resistivity for undoped MWNT-PS ( $\mathbf{a})$ and B doped MWNT-PS (O) composite films as a function of nanotube weight fraction. (c) Threshold electric field for undoped MWNT-PS (a) and B doped MWNT-PS (०) composites as a function of nanotube weight fraction.

Figure 4 Emission current density versus applied electric field for (a) undoped and (b) B doped MWNT-PS composites for different nanotube concentrations: $11.1 \%(\bullet), 14.3 \%(\mathrm{X}), 20 \%(\mathbf{\Delta}), 25 \%$ (O) and $33.3 \%(\square)$.

Figure 5 Emission site density maps made over a $3 \mathrm{~mm}^{2}$ area from a $20 \%$ B doped MWNT-PS composite at applied fields of (a) $5 \mathrm{~V} / \mu \mathrm{m}$, (b) $6 \mathrm{~V} / \mu \mathrm{m}$, (c) $7 \mathrm{~V} / \mu \mathrm{m}$ and (d) $8 \mathrm{~V} / \mu \mathrm{m}$. The maps are taken over the same area. 
Figure 6 Variation of emission current over duration of 50 hours for a $20 \%$ MWNT-PS film at applied fields of $3.2 \mathrm{~V} / \mu \mathrm{m}(\mathrm{O})$ and $4 \mathrm{~V} / \mu \mathrm{m}(\boldsymbol{\square})$.

Figure 7 (a) Higher magnification SEM image of a group of nanofibres, orientated with each other. The nanofibres have a diameter of about $0.3 \mu \mathrm{m}$ with rounded tips. (b) First ( $\bigcirc$ ) and second ( $\boldsymbol{\square}$ ) field emission I-E characteristics for the nanofibre sample. The threshold field (for $1 \mathrm{nA}$ ) can be seen as 5 $\mathrm{V} / \mu \mathrm{m}$ and not all data points are shown for clarity.

Figure 8 (a) Schematic cross section of an unetched laser crystallized silicon gated emitter. (b) Large area atomic force microscope image of a laser crystallized Si nanotip array

Figure 9 Scanning electron microscope image of an Si nanotip arrays (a) unetched and reactive ion etched for (b) 7 minutes, (c) 14 minutes and (d) 21 minutes. 


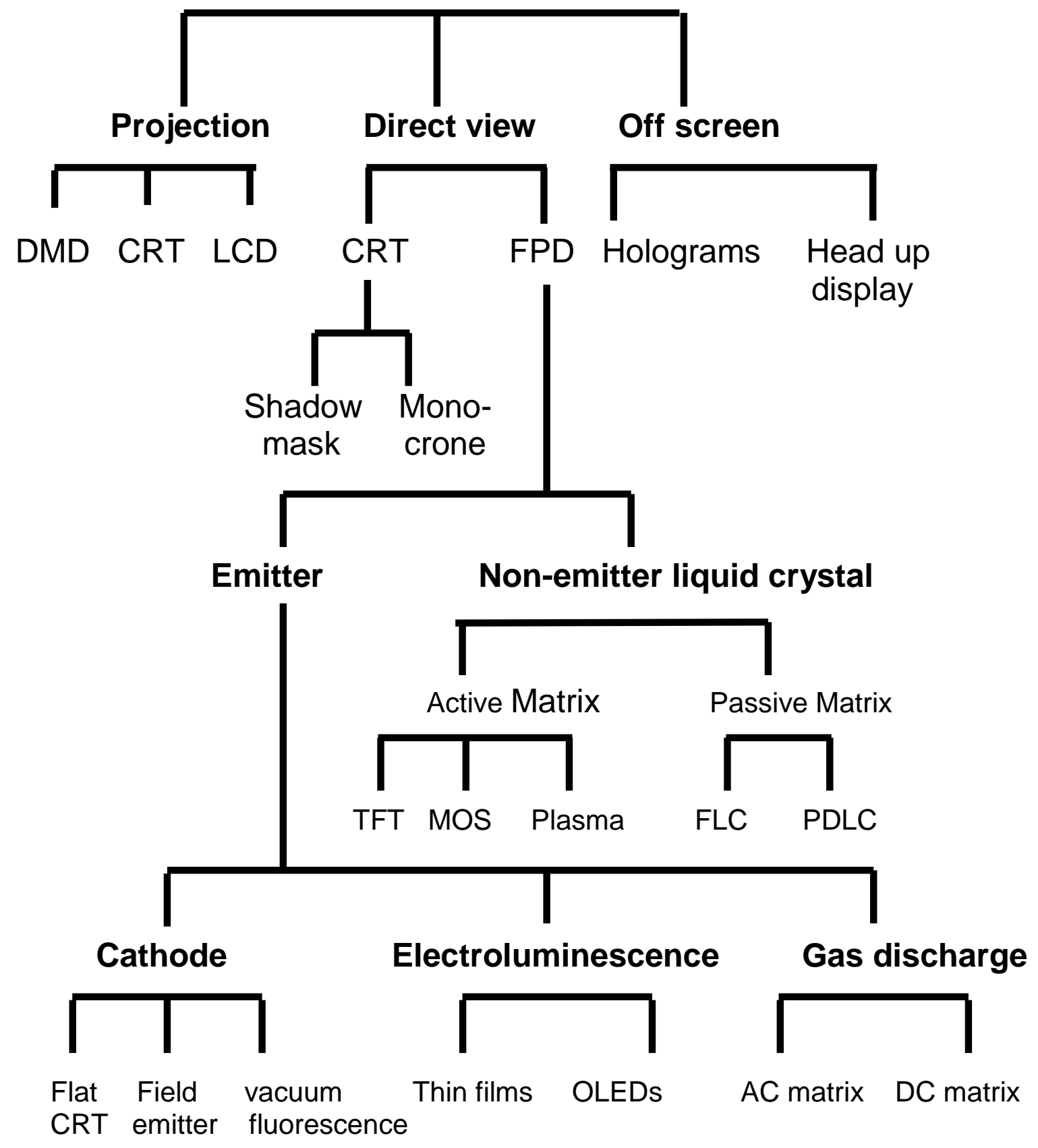

Silva et al. Figure 1 

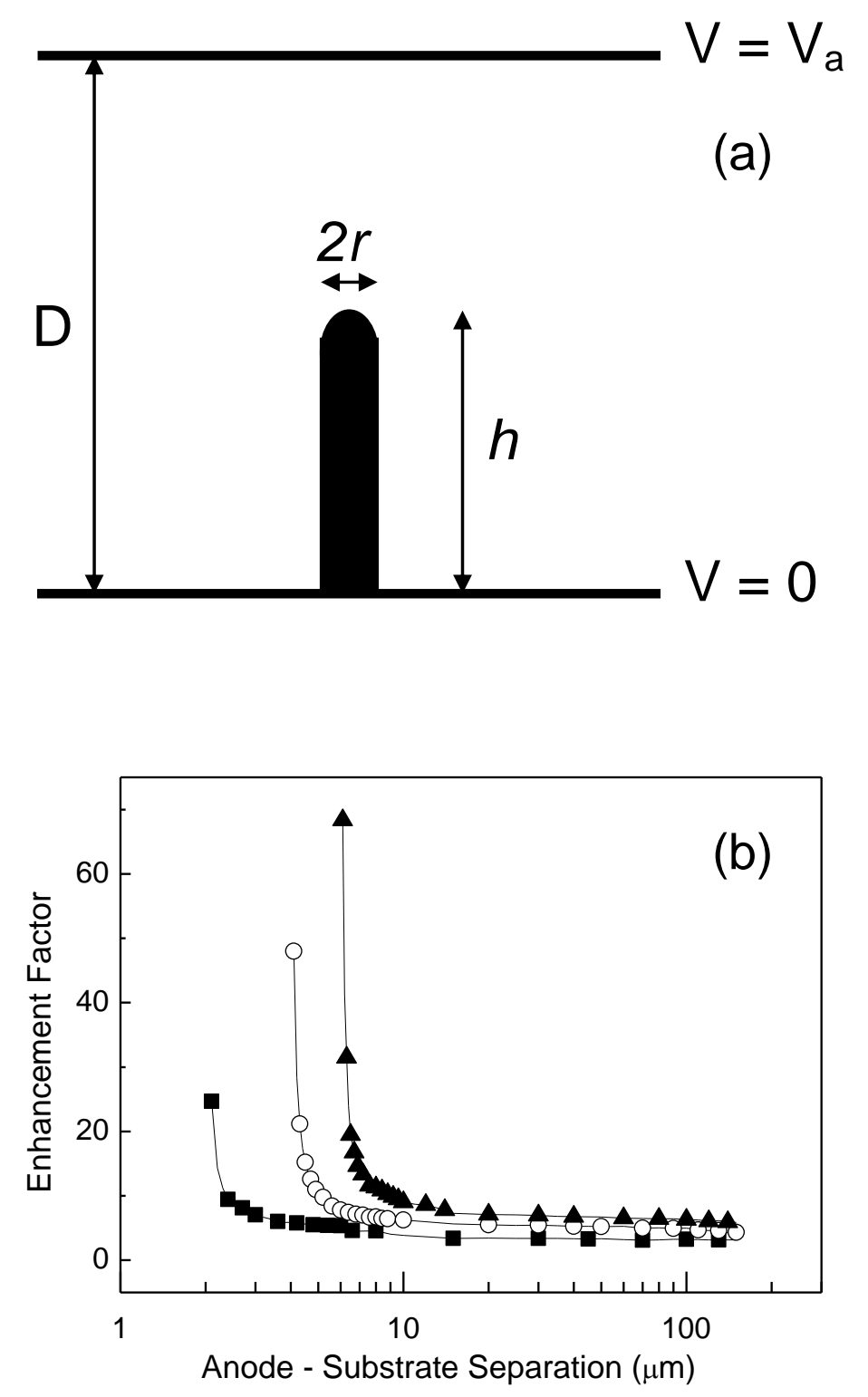

Silva et al. Figure 2 

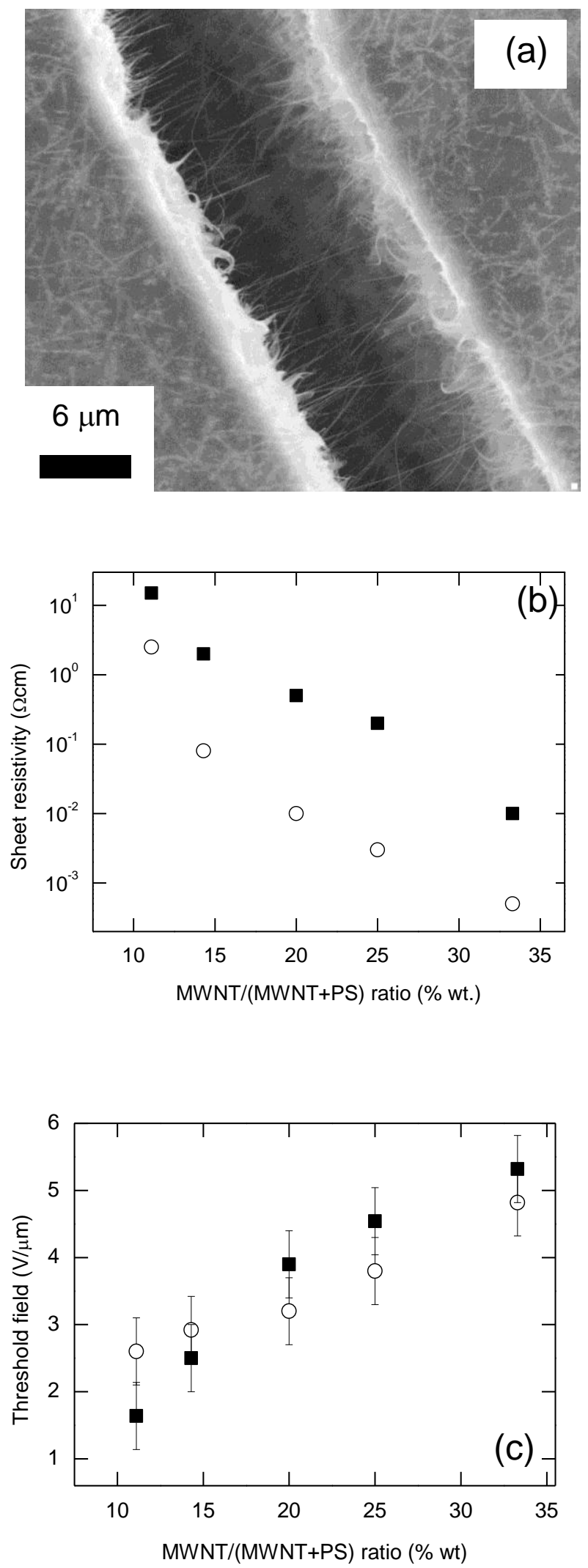

Silva et al. Figure 3 

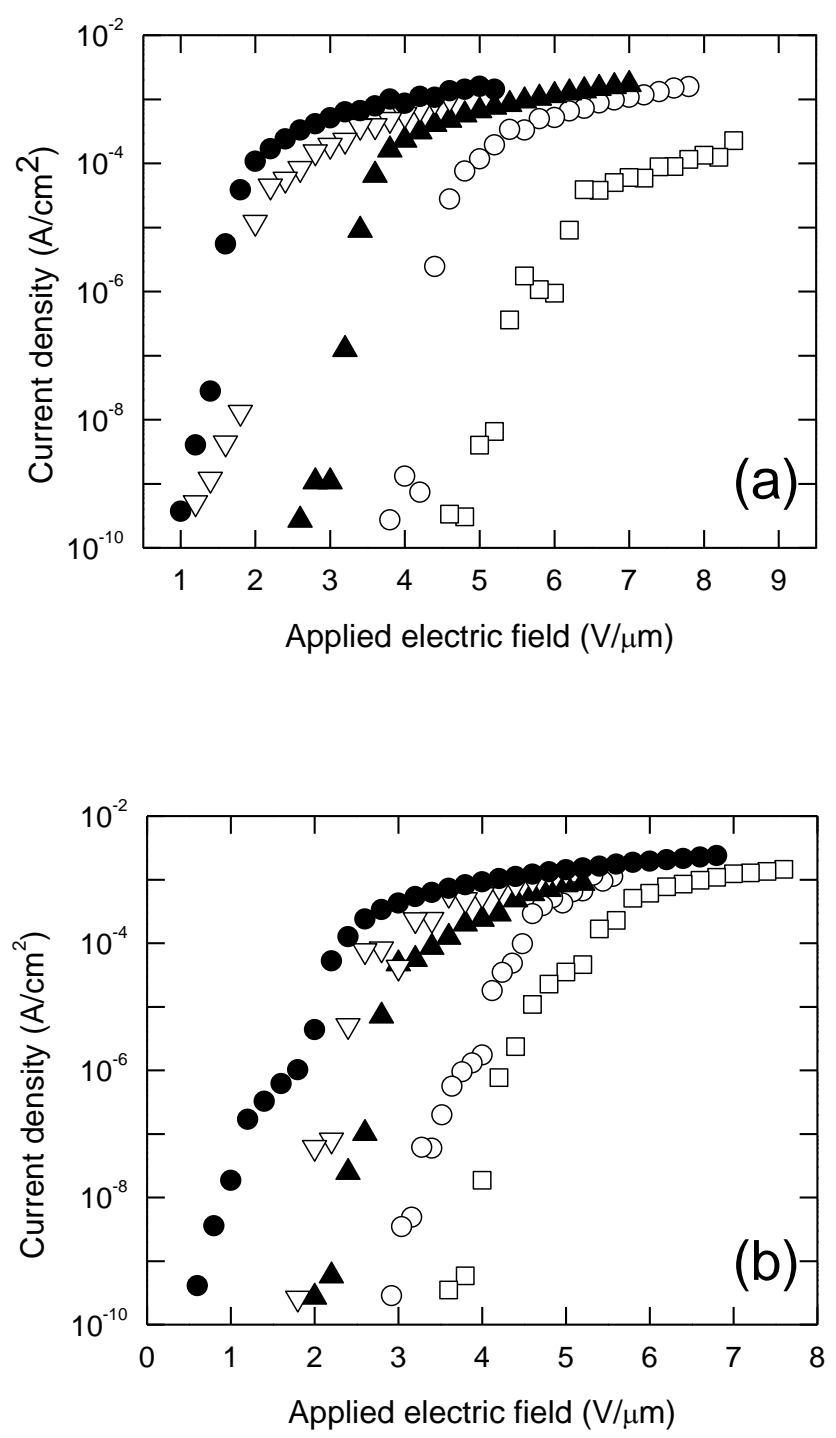

Silva et al. Figure 4 

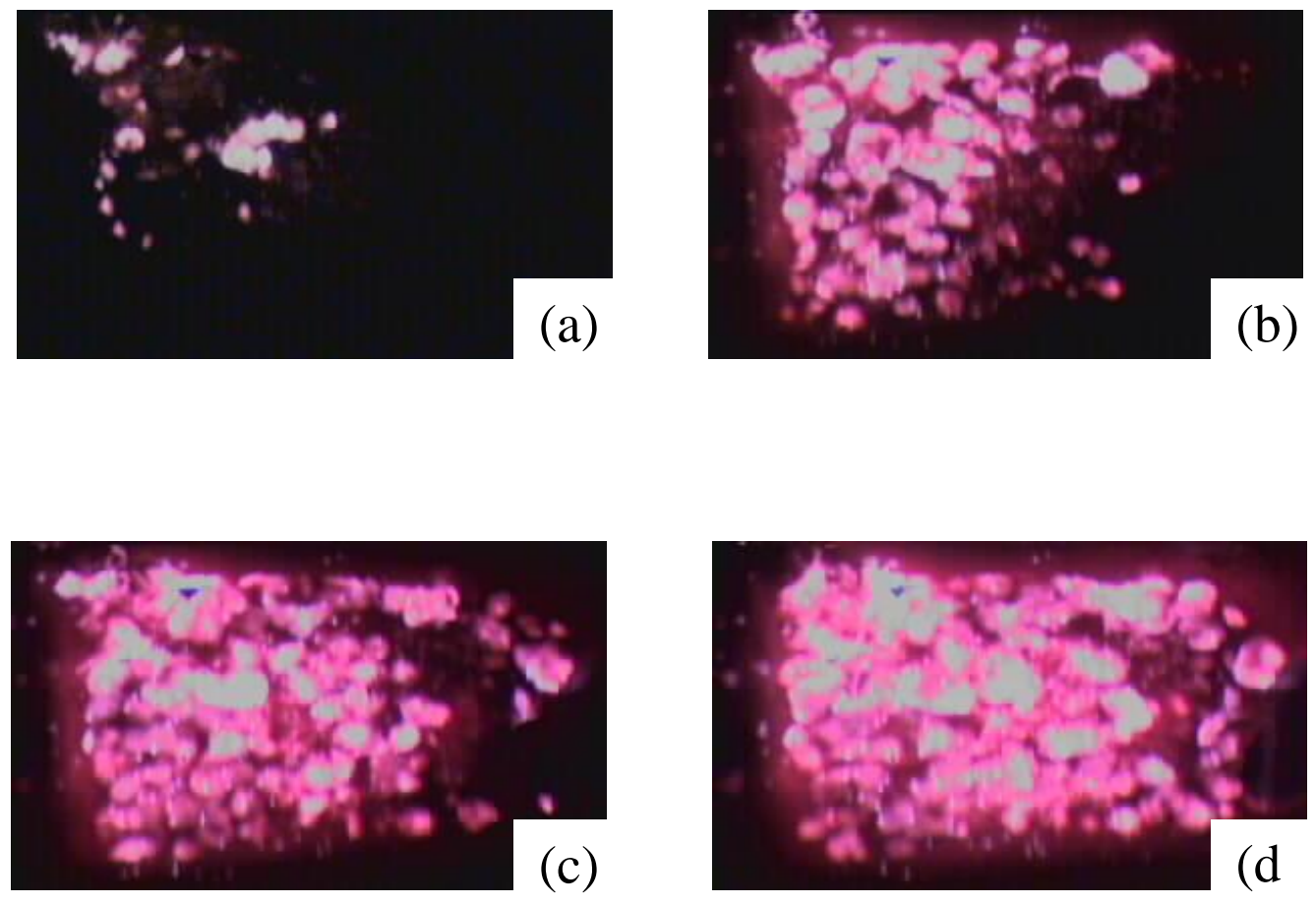

Silva et al. Figure 5 


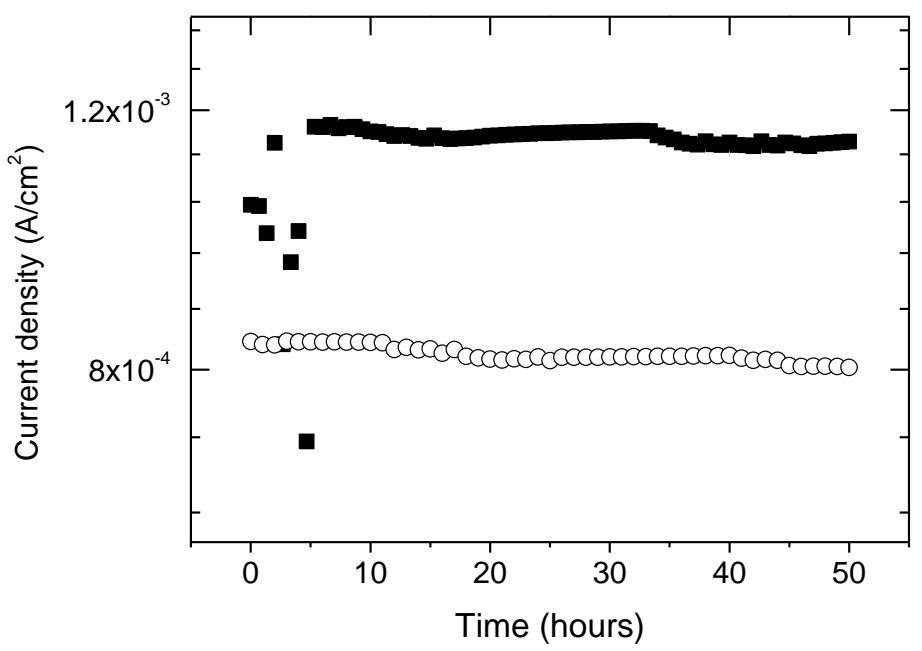

Silva et al. Figure 6 
(a)
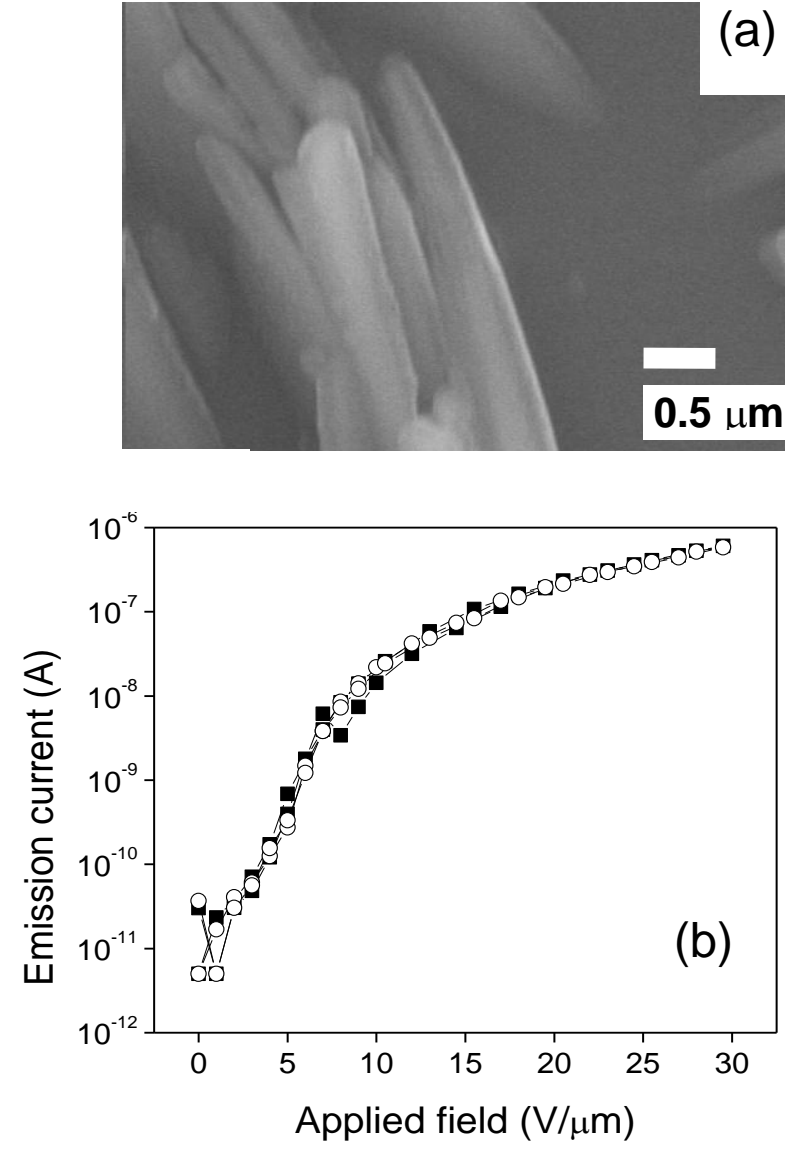

Silva et al. Figure 7 


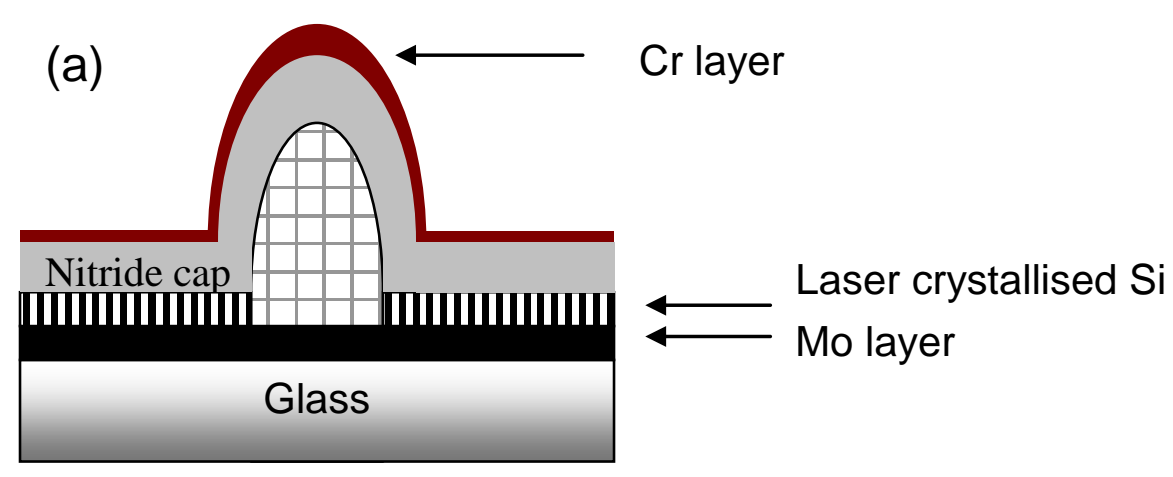

(b)

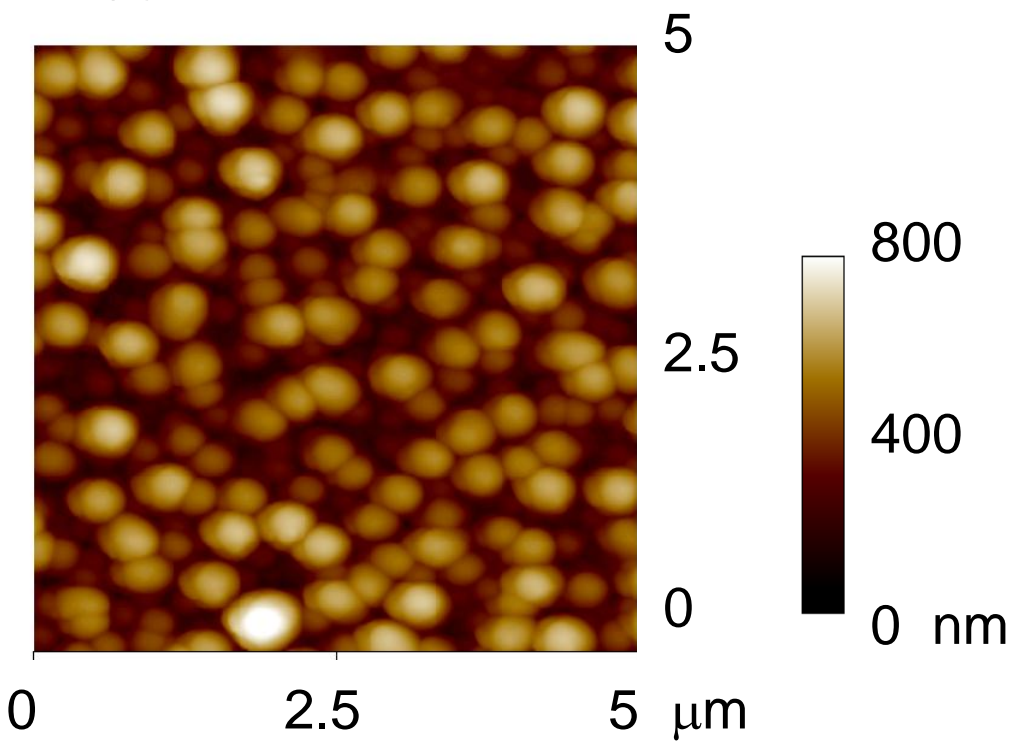

Silva et al. Figure 8 

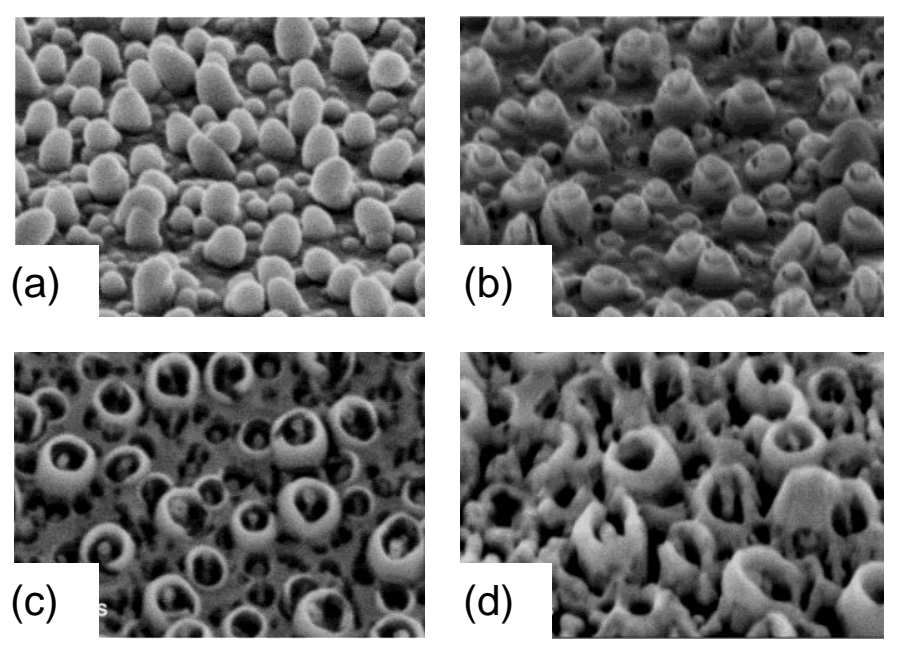

Silva et al. Figure 9 